\title{
Dilute Nitride Nanowire Lasers Based on a GaAs/GaNAs Core/Shell Structure
}

Shula Chen, Mattias Jansson, Jan Eric Stehr, Yuqing Huang, Fumitaro Ishikawa, Weimin

Chen and Irina Buyanova

\section{Journal Article}

\section{Tweet}

N.B.: When citing this work, cite the original article.

Original Publication:

Shula Chen, Mattias Jansson, Jan Eric Stehr, Yuqing Huang, Fumitaro Ishikawa, Weimin Chen and Irina Buyanova, Dilute Nitride Nanowire Lasers Based on a GaAs/GaNAs Core/Shell Structure, Nano letters (Print), 2017. 17(3), pp.1775-1781.

http://dx.doi.org/10.1021/acs.nanolett.6b05097

Copyright: American Chemical Society

http://pubs.acs.org/

Postprint available at: Linköping University Electronic Press

http://urn.kb.se/resolve?urn=urn:nbn:se:liu:diva-136051

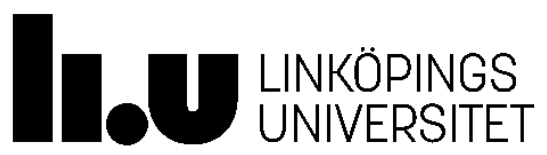




\section{Dilute Nitride Nanowire Lasers Based on a}

\section{GaAs/GaNAs Core/Shell Structure}

Shula Chen, ${ }^{\dagger, *}$ Mattias Jansson,,$^{\dagger}$ Jan E. Stehr,${ }^{\dagger}$ Yuqing Huang,,$^{\dagger}$ Fumitaro Ishikawa, ${ }^{\ddagger}$ Weimin M. Chen, ${ }^{\dagger}$ and Irina A. Buyanova ${ }^{\dagger, *}$

${ }^{\dagger}$ Department of Physics, Chemistry and Biology, Linköping University, 58183, Linköping, Sweden

¥ Graduate School of Science and Engineering, Ehime University, Matsuyama 790-8577, Japan

KEYWORDS: Nanowire lasers, dilute nitride, GaAs/GaAsN, core/shell structure.

ABSTRACT: Nanowire (NW) lasers operating in the near-infrared spectral range are of significant technological importance for applications in telecommunications, sensing and medical diagnostics. So far lasing within this spectral range has been achieved using GaAs/AlGaAs, GaAs/GaAsP and InGaAs/GaAs core/shell NWs. Another promising III-V material, not yet explored in its lasing capacity, is the dilute nitride GaNAs. In this work we demonstrate, for the first time, optically pumped lasing from the GaNAs shell of a single GaAs/GaNAs core/shell NW. The characteristic ' $S$ '-shaped pump power dependence of the lasing intensity, with the concomitant line width narrowing, is observed, which yields a threshold gain, $g_{t h}$, of $3300 \mathrm{~cm}^{-1}$ and a spontaneous emission coupling factor, $\beta$, of 0.045 . The dominant lasing peak is identified to 
arise from the $\mathrm{HE}_{21 \mathrm{~b}}$ cavity mode, as determined from its pronounced emission polarization along the NW axis combined with theoretical calculations of lasing threshold for guided modes inside the nanowire. Even without intentional passivation of the NW surface, the lasing emission can be sustained up to $150 \mathrm{~K}$. This is facilitated by the improved surface quality due to nitrogen incorporation, which partly suppresses the surface-related non-radiative recombination centers via nitridation. Our work therefore represents the first step towards development of room-temperature infrared NW lasers based on dilute nitrides with extended tunability in the lasing wavelength. 
A semiconductor nanowire naturally forms a cavity and gain medium and is, therefore, considered as an optimal building block for nano-lasers, which are technologically important for integration in highly compact optoelectronic systems. The successful demonstration of lasing in NWs has been reported for a variety of materials with a broad spectral coverage, first over nearultraviolet (UV) and visible spectral ranges and later extending to the near-infrared (NIR) spectral region [1-16]. The latter is currently attracting a growing attention as developments of efficient NIR lasers are essential e.g. for telecommunications, sensing and medical diagnostic. Current optically-pumped NIR NW lasers are most often based on GaAs/AlGaAs, GaAs/GaAsP and InGaAs/GaAs materials $[10-12,17]$. Here, the extensive research efforts were devoted to optimization of gain medium design as it determines the key parameters of laser performance, including a threshold pump density, differential gain, modulation bandwidth and thermal stability. For example, the core-shell structure of the NW cavity, where the shell has a wider bandgap than the core, is often employed to confine carriers in the core region, thereby minimizing loss due to non-radiative recombination processes and also enhancing optical amplification of cavity modes that are mainly distributed at the NW center $[10,11]$. On the other hand, the shell region can also contribute to the optical gain by incorporating radial multiple quantum wells (QWs) $[7,18,19]$. In this case, higher-order cavity modes with a ring-like light distribution become dominant in the optical output. Therefore, band engineering combined with quantum confinement effects in the NW heterostructures offer an attractive possibility to manipulate the lasing modes and also lasing wavelengths as desired for specific applications.

Flexibilities in the laser design can be further extended by utilizing III-V alloys as a gain media. One of the attractive material systems for these purposes is the GaNAs alloy. A small addition of nitrogen $(\mathrm{N})$ into GaAs causes a strong perturbation of the conduction band $(\mathrm{CB})$ states. It leads to 
admixing of the CB minimum with the upper lying extended and N-related localized states [2022], and consequently gives rise to a dramatically reduced bandgap. This is accompanied by a significant increase of the electron effective mass [23-25], which improves electron confinement in the GaNAs-based QW structures and reduces spill-out of carriers into the barrier layers, therefore improving thermal stability of laser structures [26]. Moreover we have shown [27, 28] that the presence of $\mathrm{N}$ to a certain extent improves surface properties in NW structures, likely via partial nitridation that suppresses non-radiative surface recombination known to be highly detrimental for GaAs NWs [29]. This leads to improved thermal stability of radiative efficiency, as desired for practical device applications. Given the merits above, implementing GaNAs alloys in the NW structures is expected to be advantageous for optoelectronic applications. In this study, we provide the first demonstration of lasing from a GaNAs shell co-axially grown on a GaAs core, i.e. from the GaAs/GaNAs core/shell nanowire. The lasing is attributed to the $\mathrm{HE}_{21 \mathrm{~b}}$ cavity mode and can be sustained up to $\sim 150 \mathrm{~K}$, even without intentional protection of the GaNAs surface. The utilized design with the active region in the GaNAs shell allows us to investigate lasing via ringlike cavity modes. It is also due to the fact that the growth of the high quality GaNAs crystal requires a lower growth temperature in comparison with that for GaAs, -see Methods. Therefore, the GaAs core was first grown to serve as the base for the outer GaNAs shell. We note, however, that further optimization and new development of the growth process should also allow fabrication of an inverted structure with an active GaNAs core and passivating GaAs shell, where amplification of the centrally confined fundamental modes can be achieved. The feasibility of the axial growth of dilute nitride NWs without involvement of the N-free core has recently been demonstrated in the GaNP system, where the GaNP growth was achieved by using a short seed segment of GaP [30]. The work presented here, therefore, demonstrates the potential of the GaNAs 
alloys as an active gain media and represents the first step towards realization of the GaNAs-based NW lasers.

Figure 1a shows a representative scanning electron microscopy (SEM) image of a single GaAs/GaNAs core/shell NW, which was transferred onto a gold substrate. The wire has a $180 \mathrm{~nm}-$ thick GaAs core surrounded by a 110 nm-thick GaNAs shell with the N content, [N], of $0.5 \%$, as shown schematically in Figure 1b. Before being transferred onto another substrate, all studied wires were grown on a Si (111) substrate by plasma-assisted molecular beam epitaxy (MBE) (see

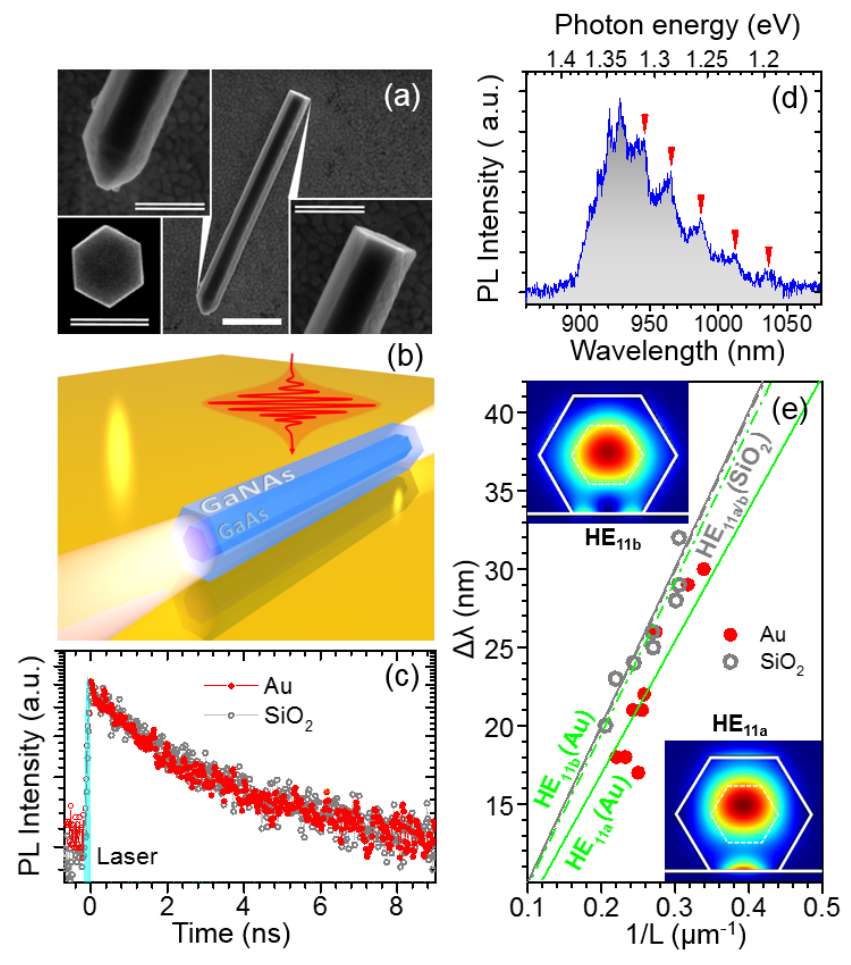

Figure 1. (a) SEM image of a single transferred GaAs/GaNAs core/shell NW. The upper-left (the lower-right) inset shows an enlarged view of the top (bottom) part of the NW. The bottom left inset represents the top-view of a free-standing NW. The solid and double scale bars represent $1 \mu \mathrm{m}$ and $400 \mathrm{~nm}$, respectively. (b) Schematic illustration of a single NW transferred on an Au substrate optically pumped by a pulsed laser. (c) $5 \mathrm{~K}$ time-resolved PL of LEs integrated within the spectral window of $1.2-1.35 \mathrm{eV}$. (d) $5 \mathrm{~K}$ PL emission from LEs at pump fluence of $0.8 \mu \mathrm{J} \mathrm{cm}^{-2}$ per pulse. (e) The mode spacing as a function of an inverse NW length, measured from a number of individual NWs on $\mathrm{Au}$ and $\mathrm{SiO}_{2}$ substrates. The insets show the profiles of the $\mathrm{HE}_{11 \mathrm{a}}$ and $\mathrm{HE}_{11 \mathrm{~b}}$ modes on the Au substrate. 
Method section for a detailed description of the NW growth). The transferred NW forms a good Fabry-Perot (F-P) cavity, which is reflected by its clear sidewalls -see inset in Figure 1a. The photoluminescence (PL) spectrum of this NW measured at $5 \mathrm{~K}$ and a low pump fluence, $P_{\text {exc }}$, is shown in Figure 1d. The spectrum is dominated by a broad asymmetric emission, which has a long extension towards low energy and a sharp high-energy cut-off. Based on our previous detailed temperature-dependent PL and PL excitation measurements [28], this emission arises from recombination of localized excitons (LEs) trapped at band tail states due to random fluctuation of the nitrogen composition. The high-energy edge of the PL spectrum at $\sim 1.39 \mathrm{eV}$ defines the bandgap energy of the GaNAs shell.

On top of the PL tail emission within the $1.2-1.35 \mathrm{eV}$ range, periodic undulations of the PL intensity are clearly observed, indicating the coupling of the LE emission to a NW cavity mode. To clarify the origin of this coupling, we measured PL spectra from several individual NWs transferred to either $\mathrm{Au}$ or $\mathrm{SiO}_{2}$ substrates (see also Supporting information I). The period of their undulation as a function of the wire length is summarized in Figure 1e (symbols). As can be seen, the mode spacing exhibits a linear relationship with the inverse NW length (L) that is typical for the F-P cavity modes, of which the mode spacing $(\Delta \lambda)$ can be expressed as [31]:

$$
\Delta \lambda=\left(\frac{\lambda^{2}}{2 L}\right)\left(n_{e f f}-\lambda \frac{d n_{e f f}}{d \lambda}\right)^{-1}
$$

Here $n_{e f f}$ and $\frac{d n_{e f f}}{d \lambda}$ are the effective index of refraction and its first order dispersion for a specific cavity mode at the wavelength $\lambda$, respectively. The term $\left(n_{e f f}-\lambda \frac{d n_{e f f}}{d \lambda}\right)$ defines the so-called group index of refraction $\left(n_{g}\right)$, which governs the propagation speed $\left(V_{g}\right)$ of the modal wave packet in the NW via $n_{g}=\frac{c}{V_{g}}$. We calculated $n_{g}$ for all the guided modes in the NW (see Supporting information II) and found that the observed periodic modulation of the PL intensity follows the 
trend of the fundamental $\mathrm{HE}_{11 \mathrm{a}} / \mathrm{HE}_{11 \mathrm{~b}}$ mode with the $n_{g}$ values of 5.33/4.63 and 4.50/4.53 for $\mathrm{Au}$ and $\mathrm{SiO}_{2}$ substrate, respectively. This can be seen from Figure 1e, where the solid (dashed) lines represent the calculated mode spacing for the $\mathrm{HE}_{11 \mathrm{a}}\left(\mathrm{HE}_{11 \mathrm{~b}}\right)$ mode in a nanowire cavity lying on a $\mathrm{SiO}_{2}$ (the grey lines) and $\mathrm{Au}$ (the green lines) substrates, respectively. The experimental data are better described by the HE11a mode, which can be attributed to the larger reflectivity, $R \sim 0.7$, of this mode at the end facets of the nanowire as compared with the HE $11 \mathrm{~b}$ mode with $R \sim 0.5$. It is not surprising to observe this fundamental F-P mode, though the LEs emission stems from the shell region. This is because the incoherent spontaneous emission from LEs at low $P_{\text {exc }}$ can be easily coupled into the $\mathrm{HE}_{11 \mathrm{a}}$ (or $\mathrm{HE}_{11 \mathrm{~b}}$ ) mode owing to its well-confined distribution across the nanowire, as shown in the insets in Figure 1e, taking as an example the NW placed on an $\mathrm{Au}$ substrate. For such wires, the question arises as to whether the observed PL emission can be affected by the surface plasmonic effect. To clarify this issue, we compared time decays of the LE emission between the NWs placed on $\mathrm{Au}$ and $\mathrm{SiO}_{2}$ substrates. The results of this comparison are shown in Figure 1c. Irrespective of the substrate materials, the decay dynamics of the integrated LE emission can be described by the same radiative lifetime of 3 ns affirming that photonic recombination prevails in the emission process. We attribute the lack of plasmonic-enhanced recombination in the wires lying on an Au substrate to the large cross-section of the NW cavity ( $400 \mathrm{~nm}$ in diameter), which substantially exceeds the scale of the plasmonic modes that is typically on the order of several tens of nanometers [32-35].

With increasing excitation power, the PL edge at $\sim 1.39 \mathrm{eV}$ blue-shifts to higher energies, indicating a gradual filling of the localized states and then extended band states by photo-excited carriers - see Figure 2a. At $\mathrm{P}_{\mathrm{exc}} \sim 13.6 \mu \mathrm{J} \mathrm{cm}^{-2}$ per pulse, a narrow line appears at $1.412 \mathrm{eV}$ (876 $\mathrm{nm}$ ) on the high energy shoulder of the emission spectrum. This line drastically gains its intensity 
with increasing $\mathrm{P}_{\mathrm{exc}}$ and eventually dominates in the PL spectra, as can also be seen from the mapping of the normalized PL intensity as a function of $\mathrm{P}_{\mathrm{exc}}$ given in Figure $2 \mathrm{~b}$. With increased pump fluence, the intensity of broad spontaneous emission background shows a gradual saturation i.e. the PL clamping, whereas the integrated intensity of the narrow line ( $\left.\mathrm{I}_{\text {int }}\right)$ is found to exhibit an apparent 'S'-shape dependence accompanied by narrowing of the PL linewidth (Figure 2c), which provides the evidence that the monitored radiative recombination evolves through three different phases, namely (i) spontaneous emission, (ii) amplified spontaneous emission, and finally (iii)
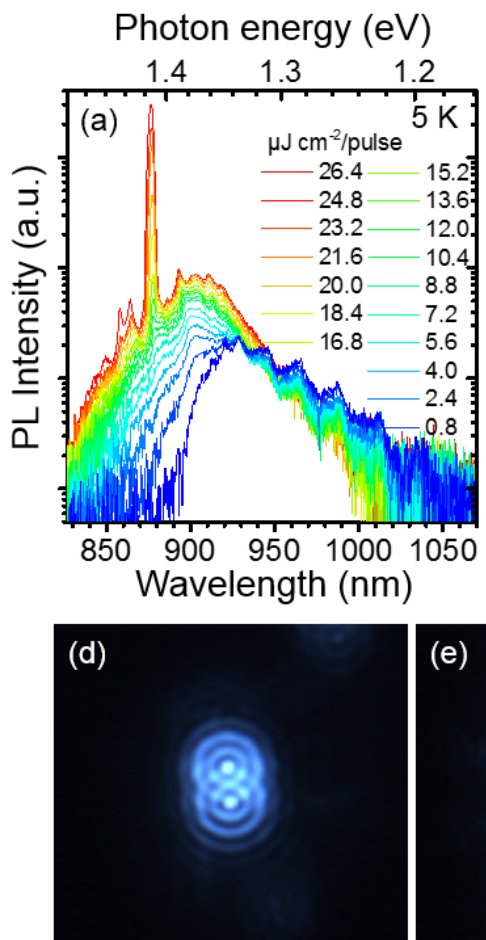
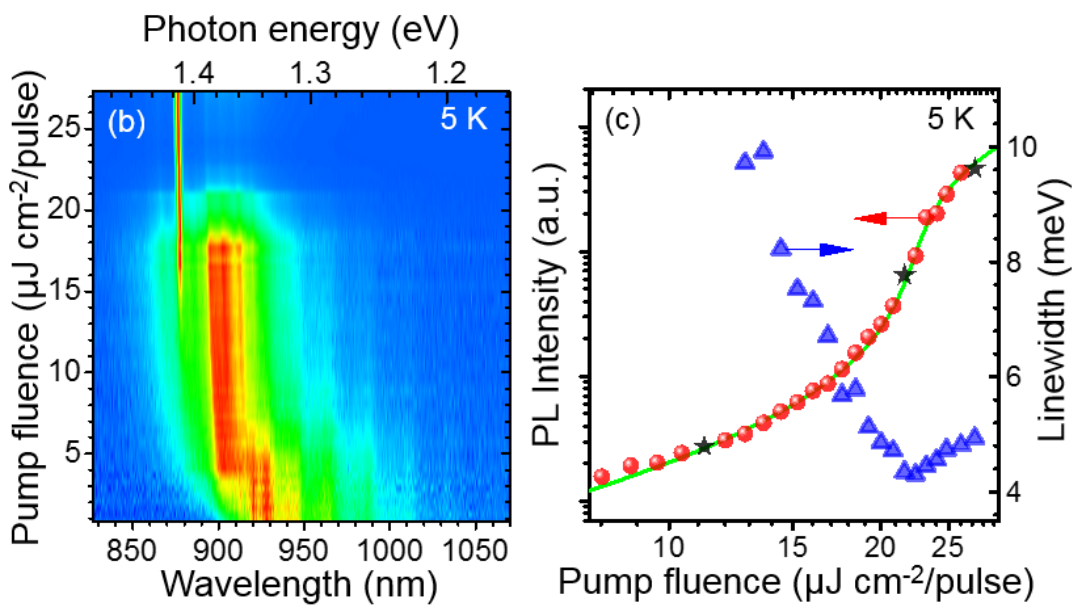
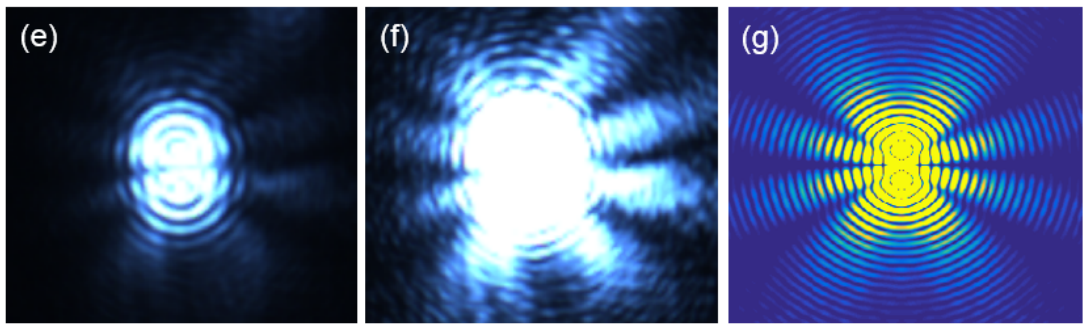

Figure 2. (a) Power dependent PL spectra at $5 \mathrm{~K}$. (b) Normalized PL intensity as a function of pump fluence and emission wavelength. (c) Double logarithmic plot of the lasing line intensity at $876 \mathrm{~nm}$ (the red dots) versus a pump fluence. The corresponding line width is shown by the blue triangles. The black stars denote the conditions used to obtain the far-field images of the emitting NWs shown in (d)-(f). The power dependence of the PL intensity fitted by a rate equation analysis is given by the green line. (d-f) Far-field images of the PL emission from a single NW under the pump fluences of 11.2,21.6, and $27.2 \mu \mathrm{J} \mathrm{cm}^{-2}$ per pulse, respectively. (g) Simulated lasing pattern by considering two coherent emitters at the NW ends, the simulation profile is similar to the observed interference pattern in (f). 
lasing. These phase changes are also visualized by the far field images of the emitted light shown in Figure 2d-f. In the spontaneous PL regime, with $\mathrm{P}_{\text {exc }}$ below $\sim 16.0 \mu \mathrm{J} \mathrm{cm}^{-2}$ per pulse, the PL peak intensity increases linearly with the laser excitation. From the PL image of the NW at $P_{\text {exc }}=$ 11.2 $\mu \mathrm{J} \mathrm{cm}^{-2}$ per pulse shown in Figure $2 \mathrm{~d}$, two bright spots are observed. This proves that the spontaneous emission primarily escapes from the two end facets of the nanowire and, in turn, implies the good quality of the NW cavity. At higher powers (e.g. $P_{\text {exc }}=21.6 \mu \mathrm{J} \mathrm{cm}^{-2}$ per pulse) (see Figure 2e), the PL output rapidly increases and a dotted pattern due to the constructive interference of the emission radiated from the two ends of the NW cavity is formed. Within this regime of the amplified spontaneous emission, a super-linear power dependence of the PL intensity on $P_{\text {exc }}$ and an appreciable narrowing of the PL linewidth are observed. Finally lasing is achieved as $P_{\text {exc }}$ exceeds $\sim 23 \mu \mathrm{J} \mathrm{cm}{ }^{-2}$ per pulse. Now the far-field optical image of the emitting NW exhibits characteristic interference-induced radiant fringes, see Figure 2f, as commonly observed for lasing NWs $[10,17,36,37]$. We simulate this lasing pattern by modelling both NW ends as two coherent emitters separated by the wire length [36] (see Supporting information). The result is shown in Figure $2 \mathrm{~g}$, which is quite similar to the observed far-field pattern of the emitted light. The power dependence in the lasing regime returns to linear-like, accompanied by an increasing linewidth due to a shorter lifetime of the stimulated emission [38]. By modelling the measured $\mathrm{I}_{\text {int }}\left(\mathrm{P}_{\mathrm{exc}}\right)$ dependence with the aid of the rate equation analysis (see Supporting information III\&IV), the threshold gain $\left(g_{t h}\right)$ and the coupling factor $(\beta)$ of spontaneous emission are derived as being 3300 $\mathrm{cm}^{-1}$ and 0.045 respectively. The results of the modelling with these parameters are shown by the green solid line in Figure 2c and are in excellent agreement with the experimental data.

Next, calculations of the theoretical threshold gain for all guided modes in the NW cavity were performed, to identify the origin of the observed lasing peak. The optical mode with the lowest 
$g_{t h}$ becomes the most favored for lasing as pump-induced gain increases. The criterion for lasing requires that the round trip gain of propagating light should exceed the total cavity loss i.e. $R^{2} e^{2 L\left(\Gamma g_{t h}-\alpha_{i}\right)}=1$. Here, $R$ is the reflectivity of the NW end facets, $\Gamma$ and $\alpha_{i}$ represent the mode confinement factor and internal propagation loss of the cavity mode, respectively. This expression allows us to estimate the theoretical $g_{t h}$ as $g_{t h}=\left(\frac{1}{\Gamma}\right)\left[\alpha_{i}+\left(\frac{1}{2 L}\right) \ln \left(\frac{1}{R^{2}}\right)\right]$, as discussed in detail in Supporting information V. The results of the performed calculations are presented in Figure 3a and show that, at the lasing wavelength of $876 \mathrm{~nm}$, the $\mathrm{HE}_{21 \mathrm{~b}}$ mode has the lowest $g_{t h}$ among all guided modes in the nanowire cavity with a diameter ranging between 360 and $450 \mathrm{~nm}$. The $g_{t h}$

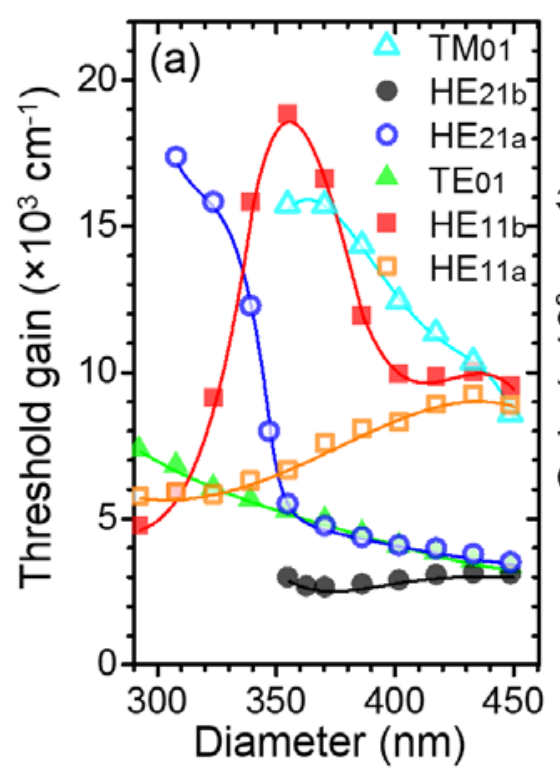

Photon energy (eV)

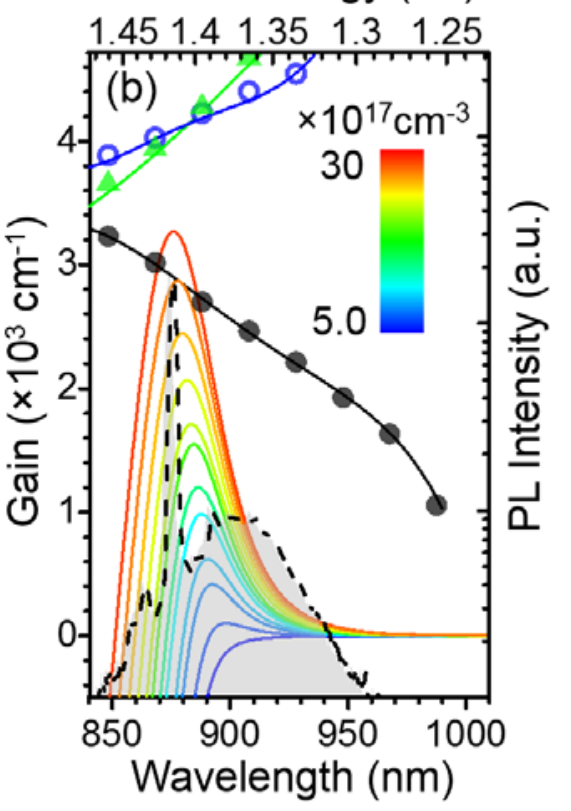

Figure 3. (a) Threshold gain calculated for the lasing emission at $876 \mathrm{~nm}$ as a function of the NW diameter for the specified guided modes. (b) Threshold gain calculated for the $\mathrm{HE}_{21} \mathrm{~b}, \mathrm{TE}_{01}$, and $\mathrm{HE}_{21 \mathrm{a}}$ modes for a nanowire with a diameter of $400 \mathrm{~nm}$ and over the emission wavelength range of $840-1000 \mathrm{~nm}$ (the three upper curves). The solid lines are the material gain spectra provided by the population of the photo-excited carriers in the GaNAs shell as a function of the carrier density. The lasing spectrum of the NW (the black dashed curve) is also shown for comparison. 
in our NWs with a diameter of $400 \mathrm{~nm}$ has the theoretical value of $\sim 2850 \mathrm{~cm}^{-1}$, which is comparable with $3300 \mathrm{~cm}^{-1}$ derived from the power dependent measurements. This value is higher than those previously reported for InGaAs/GaAs and GaAs/AlGaAs-based NW lasers [10, 12, 39] with the NW core as the active gain medium, i.e. $g_{t h} \sim 1000-2000 \mathrm{~cm}^{-1}$. The observed higher $g_{t h}$ value in our NW laser can be accounted for by a large density of the localized tail states due to alloy disorder in the GaNAs shell that needs to be saturated prior to achieving sufficient population of photo-excited carriers for lasing. Other sources that add to the higher threshold in our structures include the light absorption by Au and also light scattering at the imperfect NW end facet, e.g. the pyramid-like top part of the wire as shown in the SEM image (see Figure 1a).

The lowest $g_{t h}$ possessed by the $\mathrm{HE}_{21 \mathrm{~b}}$ mode implies that it is the most favorable mode to gain optical amplification as the pumping fluence increases. In Figure $3 b$, the $g_{t h}$ was calculated for a NW diameter of $400 \mathrm{~nm}$ and over the emission wavelength range of $840-1000 \mathrm{~nm}$. Three modes with the lowest $g_{t h}$, i.e. related to the $\mathrm{HE}_{21 \mathrm{~b}}$, TE01, and $\mathrm{HE}_{21 \text { a }}$ modes, are shown by the three upper curves in Figure $3 \mathrm{~b}$. The material gain provided by the population of the photo-excited carriers in the GaNAs shell starts to develop near $1.39 \mathrm{eV}$ at the carrier density $\left(n_{c}\right)$ of $\sim 6.0 \times 10^{17} \mathrm{~cm}^{-3}$ - see the solid lines in Figure $3 \mathrm{~b}$. It continually grows with $P_{\text {exc }}$ and reaches the threshold line defined by the $\mathrm{HE}_{21 \mathrm{~b}}$ mode at $n_{c}$ of $\sim 2.80 \times 10^{18} \mathrm{~cm}^{-3}$. The crossing of the gain profile occurs at the wavelength of $878 \mathrm{~nm}$, which is in excellent agreement with the observed lasing wavelength of $876 \mathrm{~nm}$, as shown by the overlapped lasing spectrum (the black dashed curve).

The identification of the $\mathrm{HE}_{21 \mathrm{~b}}$ mode is further corroborated by the polarization-resolved PL measurements summarized in Figure 4a. It is found that the lasing emission is strongly polarized along the NW axis, as can also be seen from the inset in Figure 4a where the azimuthal dependence of the lasing emission is shown (the green dots). The observed dependence is in good agreement 


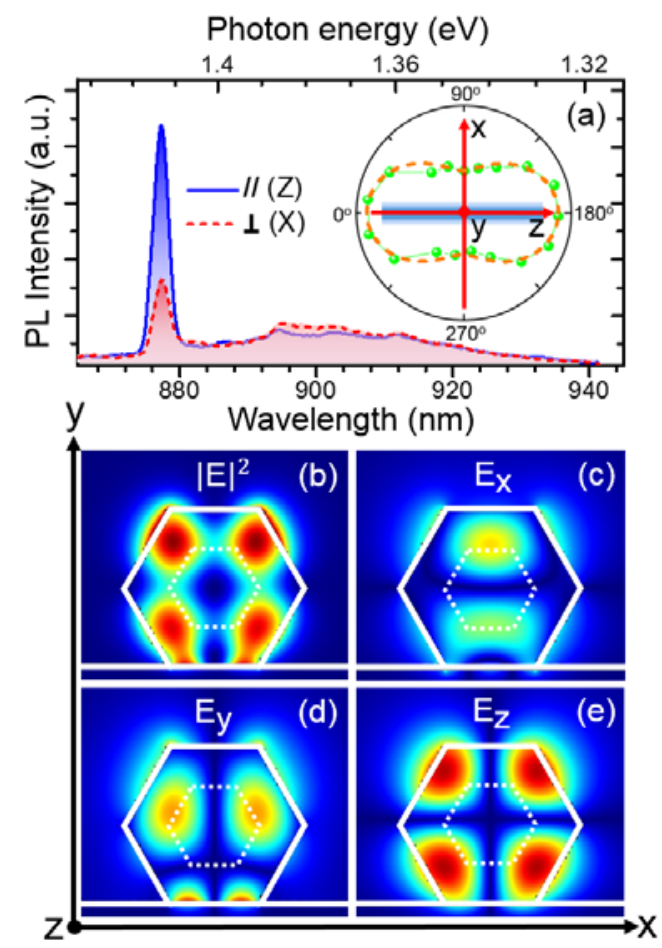

Figure 4. (a) Polarization-dependent PL spectra of the lasing NW. $\perp$ (//) denotes the polarization direction perpendicular (parallel) to the NW axis. The polarization-dependent PL intensity of the lasing line at $876 \mathrm{~nm}$ is displayed by the polar plot in the inset. (b-e) Mode intensity and its polarization along $\mathrm{x}, \mathrm{y}$ and $\mathrm{z}$ axis for the $\mathrm{HE}_{2} 1 \mathrm{~b}$ mode.

with the far-field polarization pattern for the $\mathrm{HE}_{21 \mathrm{~b}}$ mode (the dashed line in the inset of Figure 4a). To gain further understanding on why the $\mathrm{HE}_{21 \mathrm{~b}}$ mode dominates in the studied structures, its distributions in electric field intensity $|E|^{2}$ and the $\mathrm{E}_{\mathrm{x}}, \mathrm{E}_{\mathrm{y}}$ and $\mathrm{E}_{\mathrm{z}}$ components across the NW crosssection are visualized in Figure $4 \mathrm{~b}-\mathrm{e}$. It is apparent that the $\mathrm{HE}_{21 \mathrm{~b}}$ mode has the maximum intensity within the GaNAs shell and, therefore, has optimal geometric overlap with the gain medium, which enhances the light-matter interaction and ensures amplification of spontaneous emission. Similar results on lasing via 'doughnut'-like TE01 mode was recently reported in radial core/shell NWs with the optically active shell of embedded multiple quantum wells [18]. 


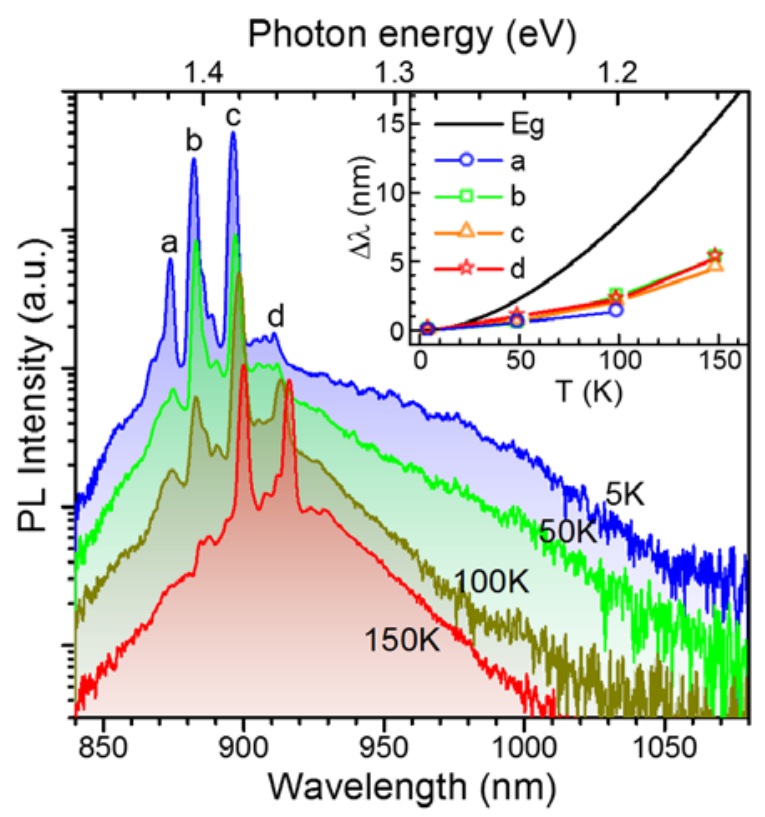

Figure 5. Temperature-dependent lasing emission from $5 \mathrm{~K}$ to $150 \mathrm{~K}$. Multiple lasing lines labeled as 'a'-' $d$ ' arise from a set of different order modes of the lasing mode. The relative wavelength shift of the bandgap (the solid line) and lasing modes (symbols) versus temperature are shown in the inset. The PL spectra were vertically shifted for clarity.

The lasing emission from the GaNAs shell can be sustained up to $\sim 150 \mathrm{~K}$, as shown in Figure 5. With rising temperature, all lasing modes gradually shift toward longer wavelengths due to a thermal variation of the refractive index [40,41]. Since the temperature-induced bandgap reduction occurs faster than the modal shift, as reflected by their relative wavelength changes shown in the inset of Figure 5, the sharp lasing lines at the higher energy side quench fast with temperature with a new mode emerging at a lower energy. A further increase of temperature leads to an overall quenching of the lasing modes due to thermally accelerated non-radiative recombination, which reduces the material gain below the threshold. The measured temperature dependence of the pump threshold $\left(P_{t h}\right)$ can be described by an exponential function $P_{t h} \propto e^{\frac{T}{T_{0}}}$, with a characteristic temperature of $T_{0} \approx 90 \pm 10 \mathrm{~K}$ (see Supporting information VI). This value, though lower, is close to the reported $T_{0}$ of $100-133 \mathrm{~K}$ in GaAs-based lasers [11, 42]. It is well known that NW structures with a high surface-to-volume ratio suffer from detrimental surface- 
related non-radiative recombination that degrades their radiative efficiency and limits their practical device application. Therefore, lasing at elevated temperatures (e.g. room temperature) was achieved only in the core/shell NW structures with a passivating outer shell with a larger bandgap $[10,12,18,42]$. The GaAs/GaNAs core/shell NWs in our study still exhibits the capability of lasing up to $\sim 150 \mathrm{~K}$ without any intentional surface treatment. This is facilitated by the presence of nitrogen in the shell layer, as revealed by our earlier temperature-dependent time resolved PL measurements $[27,28]$ that showed prolonged carrier lifetime in the shell layer due to suppression of non-radiative surface states, most likely via partial surface nitridation. We, therefore, expect that lasing can be extended to room temperature by further protecting the GaNAs layer in the GaAs/GaNAs core/shell structures, e.g. by a thin (Al)GaAs cap. Using GaNAs as an active material in such structures, which utilize radial lasing modes, may have an advantage of increased tunability in the bandgap energy without employing quantum confinement effects in complicated multishell nanowires with embedded radial multiple QWs, which are known to exhibit a higher threshold gain (e.g. of $5200 \mathrm{~cm}^{-1}[18]$ ) due to partial overlap between the electric field of the lasing mode and the gain medium.

In summary, we have demonstrated the lasing emission from a novel GaAs/GaNAs core/shell NWs. The threshold gain and spontaneous emission coupling factor were determined as being $g_{t h}=3300 \mathrm{~cm}^{-1}$ and $\beta=0.045$, respectively, based on the rate equation analysis. The dominant lasing emission was found to originate from the $\mathrm{HE}_{21 \mathrm{~b}}$ cavity mode. The incorporation of $\mathrm{N}$ into GaAs partly improved the surface quality of the nanowires and facilitated the lasing up to $150 \mathrm{~K}$ without surface capping. More importantly, the strong interaction of $\mathrm{N}$ states with the conduction band states of GaAs provides an additional freedom in bandgap engineering in heterostructured 
NWs, thus opening new possibilities for the development of room-temperature NIR NW lasers based on dilute nitrides.

\section{Methods}

The investigated GaAs/GaNAs core/shell NWs were grown by plasma-assisted molecular beam epitaxy on (111) Si substrates using constituent Ga as a catalyst to assist NW growth, as discussed in detail in Ref. 43 and 44. The growth of the GaAs core was conducted in several steps. First, the GaAs core was grown via vapor-liquid-solid growth at $570{ }^{\circ} \mathrm{C}$ and under an equivalent pressure of an As4 beam of $1 \times 10^{-5}$ Torr. After $15 \mathrm{~min}$, the growth was interrupted for $15 \mathrm{~min}$ to induce crystallization of the Ga catalyst on the tip of the NWs. Then the growth was continued for another $15 \mathrm{~min}$ and the substrate temperature was gradually ramped down to $430{ }^{\circ} \mathrm{C}$, as required to achieve $\mathrm{N}$ incorporation during subsequent formation of a GaNAs shell. The total diameter of the GaAs core was around $180 \mathrm{~nm}$, as was verified by the performed transmission electron microscopy (TEM) measurements [44]. The GaNAs shell with the $\mathrm{N}$ composition of $0.5 \%$ was grown for 30 min with an ignited N plasma and having a flux of active nitrogen of $3.5 \times 10^{-3} \mathrm{ML} / \mathrm{s}$. The so-obtained GaAs/GaNAs core/shell NWs have the total diameter of $400 \mathrm{~nm}$ and an average length of $\sim 4 \mu \mathrm{m}$. They predominantly crystalized in the zinc-blende crystal structure and had only few structural defects (below 5 twins per micrometer) within the bottom part of the wire of 1-2 $\mu \mathrm{m}$, though the twin density progressively increased towards the NW top [45].

$\mu$-PL measurements were performed in a backscattering geometry in a He-flow cryostat. For these purposes, the NWs were mechanically transferred to either gold or $\mathrm{SiO}_{2}$ substrates. A modelocked Ti: sapphire pulsed laser with a repetition rate of $76 \mathrm{MHz}$ and temporal pulse with of 2 ps was used together with a $50 \times / \mathrm{NA}=0.5$ microscope objective for optical excitation. The laser 
photon energy was tuned to $1.59 \mathrm{eV}$ to excite the carriers above the GaNAs bandgap $(\sim 1.39 \mathrm{eV})$. The PL signal from the NWs was dispersed by a single grating monochromator and detected by a charge-coupled-device camera. The PL polarization was examined using a linear-polarization analyzer, which comprised of a rotatable half-wave-plate and a fixed Glan-Thompson linear polarizer.

\section{ASSOCIATED CONTENT}

\section{Supporting Information.}

Lasing emission from NWs on gold $(\mathrm{Au})$ and $\mathrm{SiO} 2$ substrates, effective index versus NW diameters, FDTD simulations of the threshold gain for different optical modes as a function of NW diameter, the rate equation analysis. This material is available free of charge via the Internet at http://pubs.acs.org

\section{AUTHOR INFORMATION}

\section{Corresponding Author}

*Irina Buyanova e-mail: iribu@,ifm.liu.se

*Shula Chen, e-mail: shula.chen@liu.se

\section{Funding Sources}

Swedish Energy Agency (Grant No. P40119-1),

Swedish Research Council (Grant No. 2015-05532).

Linköping University (Faculty Grant SFO-Mat-LiU No 2009 00971)

Japan Society for the Promotion of Science, KAKENHI (No. 16H05970 and 23686004) 


\section{ACKNOWLEDGMENT}

The authors would like to acknowledge the financial support from the Swedish Energy Agency (Grant No. P40119-1) and the Swedish Research Council (Grant No. 2015-05532). IB acknowledges financial support from the Swedish Government Strategic Research Area in Materials Science on Functional Materials at Linköping University (Faculty Grant SFO-Mat-LiU No 2009 00971). The NW growth was partly supported by KAKENHI (No. 16H05970 and 23686004) from the Japan Society for the Promotion of Science, research scholarships from the Kato Foundation for Promotion of Science, the Kurata Memorial Hitachi Science and Technology Foundation, and the Murata Science Foundation.

\section{REFERENCES}

(1) Zimmler, M. A.; Bao, J.; Capasso, F.; Müller, S.; Ronning, C. Appl. Phys. Lett. 2008, 93, 051101-3.

(2) Chu, S.; Wang, G.; Zhou, W.; Lin, Y.; Chernyak, L.; Zhao, J.; Kong, J.; Li, L.; Ren, J.; Liu, J. Nat. Nanotech. 2011, 6, 506-510.

(3) Johnson, J. C.; Choi, H.; Knutsen, K. P.; Schaller, R. D.; Yang, P.; Saykally, R. J. Nat. Mater. 2002, 1, 106-110.

(4) Duan, X.; Huang, Y.; Agarwal, R.; Lieber, C. M. Nature 2003, 421, 241.245.

(5) Gradečak, S.; Qian, F.; Li, Y.; Park, H.; Lieber, C. M. Appl. Phys. Lett. 2005, 87, 173111-3.

(6) Agarwal, R.; Barrelet, C. J.; Lieber, C. M. Nano Lett. 2005, 5, 917-920.

(7) Qian, F.; Li, Y.; Gradečak, S.; Park, H.; Dong, Y.; Ding, Y.; Wang, Z. L.; Lieber, C. M. Nat. Mater. 2008, 7, 701-706.

(8) Xiao, Y.; Meng, C.; Wang, P.; Ye, Y.; Yu, H.; Wang, S.; Gu, F.; Dai, L.; Tong, L. Nano Lett. 2011, 11, 1122-1126.

(9) Pan, A.; Zhou, W.; Leong, E. S. P.; Liu, R.; Chin, A. H.; Zou, B.; Ning, C. Z. Nano Lett. 2009, 9, 784-788.

(10) Saxena, D.; Mokkapati, S.; Parkinson, P.; Jiang, N.; Gao, Q.; Tan, H. H.; Jagadish, C. Nat. Photonics 2013, 7, 963-968. 
(11) Tatebayashi, J.; Kako, S.; Ho, J.; Ota, Y.; Iwamoto, S.; Arakawa, Y. Nat. Photonics 2015, 9, 501-505.

(12) Chen, R.; Tran, T. D.; Ng, K. W.; Ko, W. S.; Chuang, L. C.; Sedgwick, F. G.; Chang-Hasnain, C. Nat. Photonics 2011, 5, 170-175.

(13) Liu, X.; Xu, P.; Wu, Y.; Yang, Z.; Meng, C.; Yang, W.; Li, J.; Wang, D.; Liu, X.; Yang, Q. Nano Energy 2014, 14, 304-354.

(14) Yang, Q.; Jiang, X.; Guo, X.; Chen, Y.; Tong, L. Appl. Phys. Lett. 2009, 94, 101108-3.

(15) Yang, Z.; Wang, D.; Meng, C.; Wu, Z.; Wang, Y.; Ma, Y.; Dai, L.; Liu, X.; Hasan, T.; Liu, X.; Yang, Q. Nano Lett. 2014, 14, 3153-3159.

(16) Li, J.; Meng, C.; Liu, Y.; Wu, X.; Lu, Y.; Ye, Y.; Dai, L.; Tong, L.; Liu, X.; Yang, Q. Adv. Mater. 2013, 25, 833-837.

(17) Hua, B.; Motohisa, J.; Kobayashi, Y., Hara, S.; Fukui, T. Nano Lett. 2008, 9, 112-116.

(18) Saxena, D.; Jiang, N.; Yuan, X.; Mokkapati, S.; Guo, Y.; Tan, H. H.; Jagadish, C. Nano Lett. 2016, 16, 5080-5086.

(19) Stettner, T.; Zimmermann, P.; Loitsch, B.; Döblinger, M.; Regler, A.; Mayer, B.; Winnerl, J.; Matich, S.; Riedl, H.; Kaniber, M.; Abstreiter, G.; Koblmüller, G.; Finley, J. J. Appl. Phys. Lett 2016, 108, 011108-5.

(20) Buyanova, I. A.; Chen, W. M. Physics and Applications of Dilute Nitrides; Taylor \& Francis: London, 2004.

(21) Shan, W.; Walukiewicz, W.; Ager, J. W.; Haller, E. E.; Geisz, J. F.; Friedman, D. J.; Olson, J. M.; Kurtz, S. R. Phys. Rev. Lett. 1999, 82, 1221-1224.

(22) Wei, S.; Zunger, A. Phys. Rev. Lett. 1996, 76, 664-667.

(23) Hai, P. N.; Chen, W. M.; Buyanova, I. A.; Xin, H. P.; Tu, C. W. Appl. Phys. Lett. 2000, 77, 1843-1845.

(24) Tomić, S.; O'Reilly, E. P.; Klar, P. J.; Grüning, H.; Heimbrodt, W.; Chen, W. M.; Buyanova, I. A. Phys. Rev. B 2004, 69, 245305-12.

(25) Lindsay, A.; O’Reilly, E. P. Phys. Rev. Lett. 2004, 93, 196402-4.

(26) Kondow, M.; Uomi, K.; Niwa, A.; Kitatani T.; Watahiki, S.; Yazawa Y. Jpn. J. Appl. Phys. 1996, 35, 1273-1275.

(27) Chen, S. L.; Chen, W. M.; Ishikawa, F.; Buyanova, I. A. Sci. Rep. 2015, 5, 11653-9.

(28) Chen, S. L.; Filippov, S.; Ishikawa, F.; Chen, W. M.; Buyanova, I. A. Appl. Phys. Lett. 2014, 105, 253106-5. 
(29) Demichel, O.; Heiss, M.; Bleuse, J.; Mariette, H.; Fontcuberta i Morral, A. Appl. Phys.Lett. 2010, 97, 201907-3. S

(30) Sukrittanon, S.; Kuang, Y. J.; Dobrovolsky, A.; Kang, W.; Jang, J.; Kim, B.; Chen, W. M.; Buyanova, I. A.; Tu, C. W. Appl. Phys. Lett. 2014, 105, 072107-5

(31) Siegmann, A. E. Lasers; University Science Books: Sausalito, CA, 1986.

(32) Oulton. R. F.; Sorger, V. J.; Zentgraf, T.; Ma, R. M.; Gladden, C.; Dai, L.; Bartal, G.; Zhang, X. Nature 2009, 461, 629-632.

(33) Oulton. R. F.; Sorger, V. J.; Genov, D. A.; Pile, D. F. P.; Zhang, X. Nat. Photonics 2008, 2, 496-500.

(34) Ho, J.; Tatebayashi, J.; Sergent, S.; Fong, C. F.; Iwamoto, S.; Arakawa, Y. ACS Photonics 2014, 2, 165-171.

(35) Ho, J.; Tatebayashi, J.; Sergent, S.; Fong, C. F.; Ota, Y.; Iwamoto, S.; Arakawa, Y. Nano Lett. 2016, 16, 2845-2850.

(36) Van Vugt, L.; Ruhle, S.; Vanmaekelbergh, V. Nano Lett. 2006, 6, 2707-2711.

(37) Saxena, D.; Wang, F.; Gao, Q.; Mokkapati, S.; Tan, H. H.; Jagadish, C. Nano Lett. 2015, 15, $5342-5348$.

(38) Mayer, B.; Janker, L.; Loitsch, B.; Treu, J.; Kostenbader, T.; Lichtmannecker, S.; Reichert, T.; Morkotter, S.; Kaniber, M.; Abstreiter, G.; Gies, C.; Koblmuller, G.; Finley, J. J. Nano Lett. 2016, 16, 152-156.

(39) Burgess, T.; Saxena, D.; Mokkapati, S.; Li, Z.; Hall, C. R.; Davis, J. A.; Wang, Y.; Smith, L. M.; Fu, L.; Caroff, P.; Tan, H. H.; Jagadish, C. Nat. Commun. 2016, 7, 11927-7.

(40) Hua, B.; Motohisa, J.; Ding, Y.; Hara, S.; Fukui, T. Appl. Phys. Lett. 2007, 91, 131112-3.

(41) Hua, B.; Motohisa, J.; Kobayashi, Y.; Hara, S.; Fukui, T. Nano Lett. 2009, 9, 112-116

(42) Mayer, B.; Rudolph, D.; Schnell, J.; Morkötter, S.; Winnerl, J.; Treu, J.; Müller, K.; Bracher, G.; Abstreiter, G.; Koblmüller, G.; Finley, J. J. Nat. Commun. 2013, 4, 2931-4.

(43) Araki, Y.; Yamaguchi, M.; Ishikawa, F. Nanotechnology 2013, 24, 065601-7.

(44) Ahn, N.; Araki, Y.; Kondow, M.; Yamaguchi, M.; Ishikawa, F. Jpn. J. Appl. Phys. 2014, 53, 065001-5.

(45) Filippov, S.; Jansson, M.; Stehr, J. E.; Palisaitis, J.; Persson, P. O. Å.; Ishikawa, F.; Chen, W. M.; Buyanova, I. A. Nanoscale 2016, 8, 15939-15947. 
TOC figure
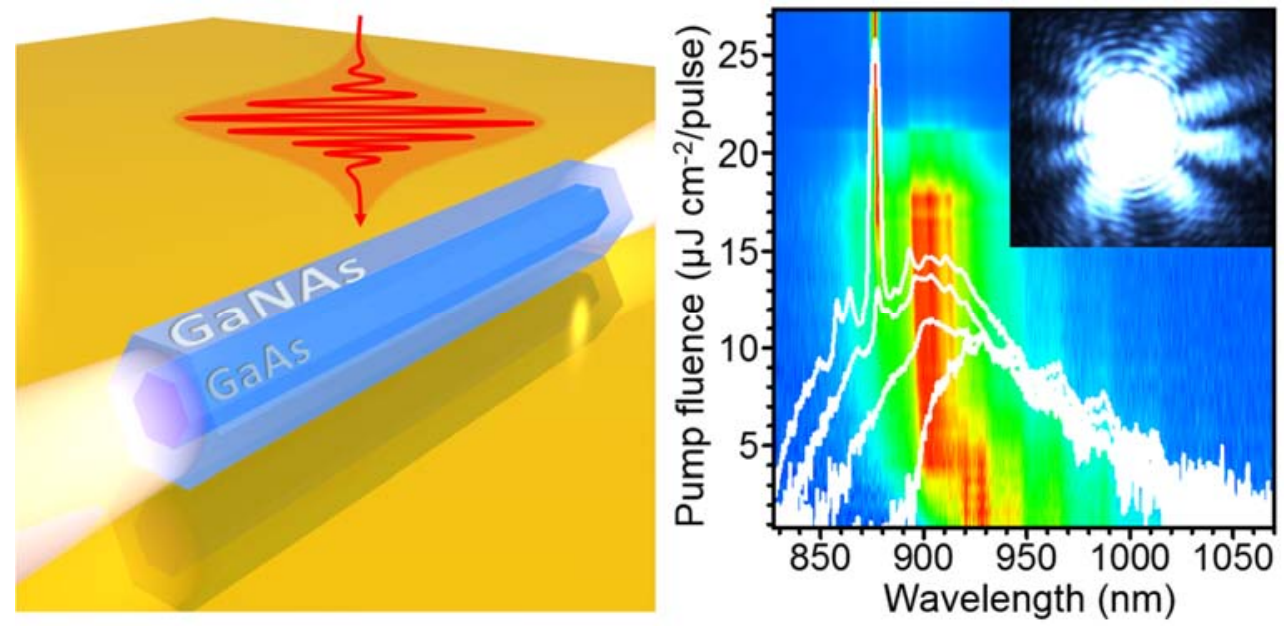